\title{
Situación laboral e impacto social de Enfermeros Graduados. Universidad del Magdalena 2007-2019.
}

\author{
Labor situation and social impact of Graduate Nurses. Magdalena University \\ 2007-2019. \\ Situação laboral e impacto social de Enfermeiros Graduados. University of \\ Magdalena 2007-2019.
}

\author{
Ediltrudis Ramos-De La Cruzl \\ Lidice Alvarez-Miño ${ }^{2}$ \\ Andreina Villa ${ }^{3}$
}

\section{Resumen}

La situación laboral de los egresados y el impacto social de la formación se constituyen en el principal insumo para que el programa adapte sus contenidos, currículos y metodologías, en pro del desarrollo las competencias profesionales que la sociedad demanda. Objetivo: Identificar la situación laboral y el impacto social de los egresados del programa de Enfermería de la Universidad del Magdalena, para el periodo comprendido entre 2007 a 2019. Materiales y métodos: Se realizó un estudio descriptivo transversal. La información, de fuente secundaria, fue aportada por la oficina de egresados de la Universidad del Magdalena, para el periodo de 2007 a 2019. La población fue de 328 graduados. Se realizaron análisis univariados y bivariados para identificar posibles diferencias significativas, $(\mathrm{Ji} 2 \mathrm{p}<0,05)$ entre las características sociodemográficas, las características laborales y de satisfacción. Resultados: La situación laboral se caracterizó por la permanencia de egresados en Colombia y en la región Caribe. Se contó con una tasa de ocupación de 69,5\%, principalmente vinculados por contrato a término fijo o por orden de prestación de servicios en el sector privado. La mayoría de los egresados se sienten satisfechos de su profesión y recomiendan el programa por su calidad. Conclusiones: Las características laborales identificadas imponen el reto institucional de generar estrategias innovadoras en nuevas pedagogías y didácticas, el desarrollo de nuevas habilidades para que los egresados tengan más opciones en el tránsito al mundo laboral. Hasta ahora se ha logrado contar con profesionales que aportan a la sociedad pero el programa debe seguirse adaptando para responder a las nuevas necesidades del entorno.

Palabras clave: Educación en Enfermería; empleo; fuerza laboral en salud; satisfacción en el trabajo; ambiente social.

Autor de correspondencia

1* Enfermera. Especialista en Cuidado Crítico, Maestría en Enfermería, Candidata a Doctora en Enfermería, Salud y Cuidado Humano. Directora del Programa de Enfermería. Universidad del Magdalena. Santa Marta, Colombia. Correo: eramos@uni$\underline{\text { magdalena.edu.co }}$ iD

${ }^{2}$ Terapeuta Ocupacional. Maestría en Salud Pública. Profesora Asociada. Programa de Enfermería. Universidad del Magdalena. Santa Marta, Colombia. Correo: lalvarezm@unimagdalena.edu.co. (iD)

${ }^{3}$ Enfermera. Especialista en Docencia Universitaria. Profesora catedrática. Programa de Enfermería. Universidad del Magdalena. Santa Marta, Colombia. Correo: avi$\underline{11 \mathrm{a} @ \text {,unimagdalena.edu.co. (iD) }}$

Recibido: 17 marzo 2020. Aprobado: 15 mayo 2020.

Para citar este artículo/ To reference this article/ Para citar este artigo/

De-la-Cruz-Ramos E, Almavez-Miño L, Villa A. Situación laboral e impacto social de Enfermeros Graduados. Universidad del Magdalena 2007-2019. Rev. cienc. cuidad. 2020; 17(3):33-45. https://doi.org/10.22463/17949831.2205 
ISSN-PRINT

$1794-9831$

E-ISSN 2322-7028

Vol. 17 No. 3

Sep - Dic 2020

Cúcuta, Colombia

\section{Abstract}

Introduction: The employment situation of the graduates and the social impact of the training constitute the main input for the program to adapt its contents, curricula and methodologies, in favor of developing the professional skills that society demands. Objective: To identify the employment situation and the social impact of the graduates of the Nursing program of the University of Magdalena, for the period from 2007 to 2019. Materials and methods: A cross-sectional descriptive study was carried out. The information, from a secondary source, was provided by the Magdalena University alumni office, for the period from 2007 to 2019. The population was 328 graduates. Univariate and bivariate analyzes were carried out to identify possible significant differences (Chi2 p <0.05) between sociodemographic characteristics with job and satisfaction characteristics. Results: The employment situation was characterized by the permanence of graduates in Colombia and in the Caribbean region. There was an occupancy rate of $69.5 \%$, mainly linked by a fixedterm contract or by order of service provision and in the private sector. Most of the graduates are satisfied with their profession and recommend the program for its quality. Conclusions: The identified work characteristics impose the institutional challenge of generating innovative strategies in new pedagogies and didactics and the development of new skills so that graduates have more options in the transition to the world of work. Until now, it has been possible to have professionals who contribute to society, but the program must continue to adapt to new needs in the environment.

Keywords: Nursing Education; Job; Health Workforce; Job Satisfaction; Social environment.

\section{Resumo}

A situação de emprego dos graduados e o impacto social do treinamento são o principal insumo para que o programa adapte seus conteúdos, currículos e metodologias, a fim de desenvolver as habilidades profissionais que a sociedade exige. Objetivo: Identificar a situação de emprego e o impacto social dos graduados do Programa de Enfermagem da Universidade Magdalena para o período de 2007 a 2019. Materiais e métodos: Foi realizado um estudo descritivo transversal. As informações, de uma fonte secundária, foram fornecidas pelo escritório de pós-graduação da Universidade Magdalena, para o período de 2007 a 2019. A população era de 328 formados. Foram realizadas análises univariadas e bivariadas para identificar possíveis diferenças significativas, (Ji2 p<0,05) entre características sociodemográficas, características de trabalho e satisfação. Resultados: A situação de trabalho foi caracterizada pela permanência de graduados na Colômbia e na região do Caribe. Havia uma taxa de emprego de $69,5 \%$, ligada principalmente por contrato a prazo ou por ordem de prestação de serviços no setor privado. A maioria dos graduados está satisfeita com sua profissão e recomenda o programa por sua qualidade. Conclusões: As características trabalhistas identificadas impõem o desafio institucional de gerar estratégias inovadoras em novas pedagogias e didáticas, o desenvolvimento de novas habilidades para que os graduados tenham mais opções na transição para o mercado de trabalho. Até agora, os profissionais têm contribuído para a sociedade, mas o programa deve continuar a ser adaptado para responder às novas necessidades do meio ambiente.

Palavras-chave: Educação de enfermagem; emprego; força de trabalho em saúde; satisfação profissional; ambiente social. 


\section{Introducción}

El impacto social de los egresados se determina desde el proceso de formación a través de los planes de estudio que las instituciones de educación superior diseñan, los cuales incorporan los conocimientos y competencias a desarrollar según requerimientos de los posibles contextos en los que se van a desenvolver (1). En el caso de los profesionales de la salud, su desempeño evidencia el impacto de su formación al transformar la salud de la población, afectando los indicadores de salud en el individuo, la familia y la comunidad en el contexto donde trabaje (2).

El contexto del ejercicio profesional está definido por los ámbitos internacional, nacional y/o local donde se puede desempeñar el graduado; los cuales, a su vez, permiten establecer la forma como el trabajo de los egresados aporta al desarrollo de las comunidades a diferente escala. Por ejemplo, pueden diseñar modelos que mejoren la salud de las poblaciones en diversos países e igualmente, su trabajo constante puede lograr que comunidades aisladas mejoren su bienestar y vida en territorios pequeños (3).

Dentro del marco de la acreditación institucional, identificar el impacto de los egresados es una forma de evidenciar el cumplimiento de la misión del programa académico (4), que en el presente trabajo aplica a la formación en enfermería. Las instituciones de educación superior (IES), deben esforzarse en comprender la sociedad con sus continuos cambios para adaptar los planes de estudio de los futuros profesionales a las demandas variables de la sociedad, si no lo hacen, se pueden estancar y aislar. En este sentido, la acreditación ayuda a dinamizar este proceso permanente de ajuste académico, desde diferentes perspectivas administrativas, organizacionales, pedagógicas, etc (5).

Los estudios de impacto de egresados surgieron frente a la necesidad de las IES de evaluar y ajustar sus programas académicos en función de las cambiantes demandas laborales en la economía globalizada (6). Según los lineamientos de acreditación del Consejo Nacional de Acreditación (CNA) en 2013(7), la alta calidad de un programa académico "se reconoce a través del desempeño laboral de sus egresados y del impacto que éstos tienen en el proyecto académico y en los procesos de desarrollo social, cultural y económico en sus respectivos entornos". El acuerdo 01 de 2018 del Consejo Nacional de Educación Superior (CESU) (8), menciona en la condición de calidad de egresados que las IES deben contar con sistemas de información que permitan ubicar a los egresados y conocer las actividades que desarrollan para asegurar la pertinencia de la oferta educativa, entre otros aspectos.

En definitiva, las características del desempeño laboral y el impacto social de los egresados son esenciales para redefinir los planes de estudio y garantizar la calidad de los programas académicos. Los aspectos que definen el impacto social y académico y la situación laboral se presentan en el cuadro 1.

Con el fin de identificar elementos para mantener actualizado y articulado el programa de enfermería a las necesidades cambiantes del contexto, se desarrolló el presente estudio, que tuvo como objetivo establecer el impacto social y la situación laboral de los egresados del programa de Enfermería de la Universidad del Magdalena, para el periodo comprendido entre 2007 a 2019. 
Cuadro 1. Elementos que constituyen el impacto social del egresado y la situación laboral.

ISSN-PRINT

1794-9831

E-ISSN 2322-7028

Vol. 17 No. 3

Sep - Dic 2020

Cúcuta, Colombia

\begin{tabular}{ll}
\hline \multicolumn{1}{c}{ Situación laboral } & \multicolumn{1}{c}{ Impacto social y académico } \\
\hline Experticia relacionada con el área de for- & Egresados del programa que forman parte de comuni- \\
mación, participación en la creación de & dades académicas reconocidas, de asociaciones cien- \\
empresa. & tíficas, profesionales, tecnológicas, técnicas o artísti- \\
& cas, y del sector productivo y financiero, en ámbitos \\
& nacional o internacional.
\end{tabular}

Correspondencia entre la ocupación y ubicación profesional de los egresados y el perfil de formación del programa. Relación del trabajo actual con el área

Egresados del programa que han recibido distinciones y reconocimientos significativos por su desempeño en la disciplina, profesión, ocupación u oficio correspondiente

Apreciación de los egresados, empleadores y usuarios externos sobre la calidad de la formación dada por el programa.*

Apreciación de los egresados acerca de la forma como el programa favorece el desarrollo del proyecto de vida

Fuente: Elaboración propia con base en Lineamientos para la acreditación de programas. CNA. Bogotá, Colombia, diciembre de 2013(7) y el documento Impacto laboral, económico y social de los graduados de la Universidad CES; 2016 (9).

*Específicamente, para este trabajo solo se consideraron los elementos aportados por los mismos egresados.

\section{Materiales y Método}

Se desarrolló un estudio descriptivo transversal, porque las variables fueron medidas una sola vez en la población, que utilizó información de fuente secundaria (10). La oficina de egresados de la Universidad del Magdalena diseñó y aplicó una encuesta electrónica enviada a la totalidad de direcciones de correo electrónico de sus bases de datos de egresados, es decir, 695 egresados a la fecha de aplicación, que contemplo el periodo comprendido entre el año 2007 y el primer semestre de 2019. Se obtuvo un total de 351 respuestas, de las cuales se eliminaron 23 registros por tener incompleto más del 50\% del instrumento. Sobre los resultados de 328 participantes (tasa de respuesta del 47\%) se hicieron los análisis estadísticos.

La encuesta aplicada por la oficina de egresados contó con 48 preguntas agrupadas en:

1. Datos sociodemográficos, con 10 ítems, como: Edad, sexo, país de origen, lugar de residencia, lugar y fecha de nacimiento, pertenencia a algún grupo poblacional, entre otros.En esta categoría las variables que se incluyeron fueron principalmente de tipo cualitativo nominal (lugares); dicotómicas (sexo) y cuantitativas (edad, año de grado).

2. Situación laboral, con 11 ítems, como: Vinculación laboral al momento de la encuesta, lugar de trabajo, área, tipo de empresa, tipo de vinculación, salario, satisfacción, tiempo de vinculación, etc. La mayoría de las variable fueron cualitativas nominales, excepto satisfacción que tuvo una escala ordinal con 4 niveles desde muy insatisfecho a muy satisfecho $y$ las variables de salario que se indagaron en rangos desde 2 SMLV hasta más de 6SMLV y los meses de desempleo que se indagaron en rangos de 1 a 6,7 a 12, 13 a 18 y 19 a 24 meses.

3. Impacto socio-académico, con 27 ítems, como: Formación de posgrado, manejo de idiomas, miembro de comunidades científicas $\mathrm{o}$ académicas, fortalezas y debilidades que considera le aportó el programa, investigación y producción, emprendimientos o empresas propias, distinciones que ha recibido en su ejercicio profesional, fortalezas y debilidades de la formación de cara al desempeño laboral, entre otros. La mayoría de las variables fueron de tipo nominal dicotómico y politómico, las demás fueron ordinales con tres opciones 
(bueno, regular, malo) para la calidad de la formación recibida y (excelente, buena, regular) para la calidad de la Universidad.

Para el análisis se consolidó y depuró la base de datos en Excel y se hicieron los análisis descriptivos univariados y bivariados $\left(\mathrm{Ji}^{2}\right)$, para identificar posibles diferencias significativas por características sociodemográficas con características laborales y satisfacción, utilizando el programa de licencia abierta JASP.

Con respecto a los aspectos éticos se declara que esta investigación se suscribió a los códigos y normas éticas vigentes que hacen relación a investigación con seres humanos, en especial a la
Resolución Número 8430 de 1993, Artículo 11. Numeral a) Investigación sin riesgo. Debido a que la información fue de fuente secundaria, suministrada por la oficina de egresados, los investigadores garantizaron a confidencialidad de la misma y el uso exclusivo de estos datos para la investigación.

\section{Resultados}

En el grupo de egresados participantes, la media de la edad fue 29,5 años (DS 4,4), con un mínimo de 22 años y un máximo de 48 . El tiempo de graduado fue en promedio de 5,2 años (DE: 2,9). Otras características se presentan en la tabla 1.

Tabla 1. Características sociodemográficas de los egresados participantes

\begin{tabular}{|c|c|c|c|}
\hline Variables & & Frecuencia & $\%$ \\
\hline \multirow[t]{3}{*}{ Sexo } & Femenino & 275 & 83,8 \\
\hline & Masculino & 50 & 15,3 \\
\hline & Sin dato & 3 & 0,9 \\
\hline \multirow[t]{6}{*}{ Edad } & $22-25$ & 71 & 21,6 \\
\hline & $26-29$ & 103 & 31,4 \\
\hline & $30-33$ & 86 & 26,2 \\
\hline & $34-37$ & 48 & 14,6 \\
\hline & 38 y más & 16 & 4,8 \\
\hline & Sin dato & 4 & 1,2 \\
\hline \multirow[t]{5}{*}{ País de Residencia } & Argentina & 3 & 0,9 \\
\hline & Brasil & 3 & 0,9 \\
\hline & Colombia & 316 & 96,4 \\
\hline & Estados Unidos & 1 & 0,3 \\
\hline & Sin dato & 5 & 1,5 \\
\hline \multirow[t]{7}{*}{ Pertenencia a algún grupo poblacional } & Ninguna & 293 & 86,3 \\
\hline & Madre Cabeza de Hogar & 14 & 4,2 \\
\hline & Grupo étnico & 5 & 1,5 \\
\hline & LGBTI & 6 & 1,8 \\
\hline & Discapacidad & 1 & 0,3 \\
\hline & Víctima del conflicto & 5 & 1,5 \\
\hline & Sin dato & 4 & 1,2 \\
\hline \multirow[t]{6}{*}{ Año de Grado } & 2007 a 2009 & 40 & 12,2 \\
\hline & 2010 a 2012 & 58 & 18 \\
\hline & 2013 a 2015 & 122 & 37 \\
\hline & 2016 a2018 & 100 & 31 \\
\hline & 2019 (1) & 2 & 0,6 \\
\hline & Sin dato & 6 & 2 \\
\hline TOTAL & & 328 & 100 \\
\hline
\end{tabular}

Fuente: Elaboración propia con base en datos de encuesta. 
ISSN-PRINT

$1794-9831$

E-ISSN 2322-7028

Vol. 17 No. 3

Sep - Dic 2020

Cúcuta, Colombia
Como se observa en la tabla 1, la mayoría de los graduados residen en Colombia y de ellos el 74\% se ha mantenido en la región Caribe del país.

Con relación a la situación laboral al momento de la encuesta, se encontró que un $30,5 \%$ de los egresados participantes manifestó estar sin trabajo y el $35,4 \%$ refirió haber estado sin trabajo por un periodo entre 1 y 6 meses. El medio más efec- tivo para conseguir trabajo fue por referencias de conocidos en un $34,5 \%$ e Internet el $19,5 \%$, la Universidad como medio para conseguir trabajo solo fue reconocida en un 5,2\%. Un 38\% trabajan en empresas privadas, el $59 \%$ consideran que el trabajo se relaciona con la formación recibida y el $62 \%$ devenga entre 2 a 4 salarios mínimos legales vigentes (Ver tabla 2).

Tabla 2. Situación Laboral de los Egresados participantes

\begin{tabular}{|c|c|c|c|}
\hline \multicolumn{2}{|l|}{ Variables } & \multirow{2}{*}{$\begin{array}{r}\text { número } \\
100\end{array}$} & \multirow{2}{*}{$\begin{array}{ll}\% & \\
& 30,5\end{array}$} \\
\hline Actualmente se encuentra laborando & No & & \\
\hline & $\mathrm{Si}$ & 226 & 68,9 \\
\hline & Sin dato & 2 & 0,6 \\
\hline \multirow{4}{*}{$\begin{array}{l}\text { El trabajo que realiza se relaciona con su } \\
\text { formación }\end{array}$} & Directamente relacionado & 193 & 58,8 \\
\hline & Indirectamente relacionado & 17 & 5,2 \\
\hline & Nada relacionado & 2 & 0,6 \\
\hline & Sin dato y Desempleados & 116 & 35,4 \\
\hline \multirow[t]{5}{*}{ Tipo de empresa en la cual labora } & Pública & 85 & 25,9 \\
\hline & Privada & 126 & 38,4 \\
\hline & Propia & 1 & 0,3 \\
\hline & Otra & 2 & 0,6 \\
\hline & Sin dato y desempleado & 114 & 34,7 \\
\hline \multirow[t]{5}{*}{ Tipo de vinculación } & Contratación por prestación de servicios & 57 & 17,4 \\
\hline & Contrato a término fijo & 59 & 18,0 \\
\hline & Contrato a término indefinido & 91 & 27,7 \\
\hline & Otro & 7 & 2,1 \\
\hline & Sin dato y Desempleados & 114 & 34,7 \\
\hline \multirow{6}{*}{ Tipo de empresa donde trabaja } & Local & 46 & 14 \\
\hline & Multinacional & 13 & 3,9 \\
\hline & Nacional & 89 & 27,1 \\
\hline & ONG & 4 & 1,2 \\
\hline & Regional & 62 & 18,9 \\
\hline & Sin dato y desempleado & 114 & 34,8 \\
\hline \multirow{6}{*}{ Ingresos mensuales } & Entre 2 y 3 SMMLV & 158 & 48,2 \\
\hline & Entre 3 y 4 SMMLV & 46 & 14 \\
\hline & Entre 4 y 5 SMMLV & 5 & 1,5 \\
\hline & Entre 5 y 6 SMMLV & 2 & 0,6 \\
\hline & Más de 6 SMMLV & 3 & 0,9 \\
\hline & Sin dato y desempleado & 114 & 34,8 \\
\hline \multirow{5}{*}{$\begin{array}{l}\text { Meses que ha estado sin trabajo desde que } \\
\text { se graduó }\end{array}$} & 1 a 6 & 116 & 35,4 \\
\hline & 7 a 12 & 38 & 11,6 \\
\hline & 13 a 18 & 4 & 1,2 \\
\hline & 19 a 24 & 9 & 2,7 \\
\hline & Sin dato & 161 & 49,1 \\
\hline TOTAL & & 328 & 100 \\
\hline
\end{tabular}

Fuente: Elaboración propia con base en datos de encuesta. 
Con respecto a los análisis bivariados, se encontró diferencia significativa entre grupos de pertenencia a un grupo poblacional (étnico, LGBTI, madre cabeza de hogar, víctima del conflicto y en situación de discapacidad) y no pertenecer a ningún grupo con encontrarse trabajando al momento de la encuesta $\left(X^{2}=7,21 ; p=0,007\right)$. No se identificó diferencia significativa para ninguna de las variables de situación laboral medidas por sexo ni edad $(\mathrm{p}>0,05)$.

Frente al grado de satisfacción percibido por los graduados los resultados se presentan distribuidos por sexo, edad y grupo poblacional en la Figura 1. No se encontró diferencia significativa en la satisfacción por edad, sexo ni pertenencia a grupo poblacional $(\mathrm{p}>0,05)$.
ISSN-PRINT

1794-9831

E-ISSN 2322-7028

Vol. 17 No. 3

Sep - Dic 2020

Cúcuta, Colombia

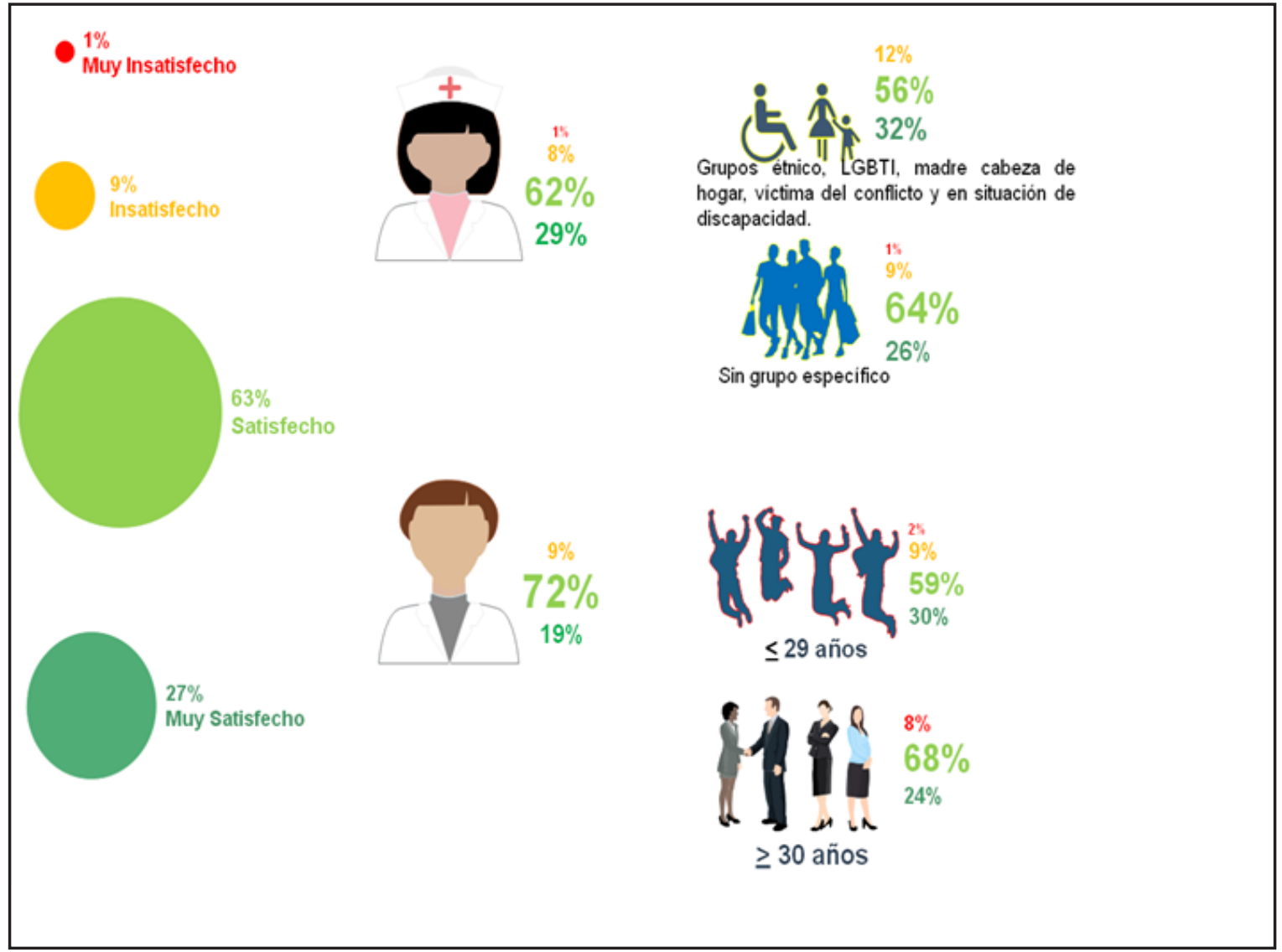

Figura 1. Distribución de los niveles de satisfacción laboral por total de participantes, sexo, pertenencia a grupos específicos y grupos de edad.

Fuente: Elaboración propia con base en los datos arrojados por la encuesta. Elaborada en EdrawInfo

Finalmente, con relación al impacto social de los graduados, se encontró que el 34\% refiere haber realizado posgrados, la mayoría especializaciones en las áreas de administración y auditoría.
Del 15\% que manejan otros idiomas, la mayoría refirió inglés, seguido de francés, italiano y portugués. (Ver tabla 3). 
Tabla 3. Impacto académico y social de los egresados

ISSN-PRINT

$1794-9831$

E-ISSN 2322-7028

Vol. 17 No. 3

Sep - Dic 2020

Cúcuta, Colombia

\begin{tabular}{|c|c|c|c|}
\hline \multicolumn{2}{|l|}{ Variables } & \multirow{2}{*}{$\begin{array}{r}\text { Frecuencia } \\
199\end{array}$} & \multirow{2}{*}{$\frac{\%}{60,6}$} \\
\hline Formación postgraduada & No & & \\
\hline & $\mathrm{Si}$ & 111 & 33,8 \\
\hline & Sin dato & 18 & 5,5 \\
\hline \multirow[t]{4}{*}{ Nivel de formación de posgrado } & Especialidad & 93 & 28,4 \\
\hline & Maestría & 17 & 5,2 \\
\hline & Doctorado & 1 & 0,3 \\
\hline & Sin dato y sin posgrado & 217 & 66,1 \\
\hline \multirow[t]{3}{*}{ Manejo de idiomas } & No & 262 & 79,9 \\
\hline & $\mathrm{Si}$ & 50 & 15,2 \\
\hline & Sin dato & 16 & 4,9 \\
\hline \multirow{3}{*}{$\begin{array}{l}\text { Miembro de comunidades científicas o aca- } \\
\text { démicas }\end{array}$} & No & 253 & 77,2 \\
\hline & $\mathrm{Si}$ & 46 & 14 \\
\hline & Sin dato & 29 & 8,8 \\
\hline \multirow{5}{*}{ Fortalezas del programa } & Asistencial & 225 & 68,6 \\
\hline & Administrativo & 36 & 11 \\
\hline & Docencia & 25 & 7,6 \\
\hline & Investigación & 13 & 4 \\
\hline & Sin dato & 29 & 8,8 \\
\hline \multirow[t]{5}{*}{ Debilidades del programa } & Asistencial & 23 & 7 \\
\hline & Administrativo & 41 & 12,5 \\
\hline & Docencia & 31 & 9,4 \\
\hline & Investigación & 202 & 61,5 \\
\hline & Sin dato & 31 & 9,4 \\
\hline \multirow{3}{*}{$\begin{array}{l}\text { Ha realizado investigaciones después de gra- } \\
\text { duarse }\end{array}$} & No & 243 & 74,1 \\
\hline & $\mathrm{Si}$ & 55 & 16,8 \\
\hline & Sin dato & 30 & 9,1 \\
\hline \multirow[t]{3}{*}{ Participación en eventos académicos } & No & 104 & 31,7 \\
\hline & $\mathrm{Si}$ & 192 & 58,5 \\
\hline & Sin dato & 32 & 9,8 \\
\hline \multirow[t]{3}{*}{ Emprendimientos o empresas propias } & No & 255 & 77,7 \\
\hline & $\mathrm{Si}$ & 40 & 12,2 \\
\hline & Sin dato & 33 & 10,1 \\
\hline \multirow[t]{3}{*}{ Distinciones y reconocimientos } & No & 249 & 75,9 \\
\hline & $\mathrm{Si}$ & 43 & 13,1 \\
\hline & Sin dato & 36 & 11 \\
\hline TOTAL & & 328 & 100 \\
\hline
\end{tabular}

Fuente: Elaboración propia

Con respecto a las fortalezas del programa, el área asistencial fue el más reconocido con un $68,6 \%$ y en cuanto a las debilidades el $61,5 \%$ refirió la investigación, lo cual podría explicar que solamente el $16,8 \%$ refiere haber realizado alguna investigación después de graduarse. Sin embargo, en cuanto a la participación en eventos el35,4\% refirió haberlo hecho como ponentes.

Por otro lado, una alta proporción de los graduados, el $84 \%$, refirió estar interesado en cursar un posgrado en la Universidad del Magdalena y un $2 \%$ ya se encuentra realizando algún posgrado en la institución. El 97,6\% de los encuestados recomendaría el programa. No se encontraron diferencias significativas para ninguna de estas variables con las características sociodemográficas (sexo, edad, pertenencia a un grupo, $\mathrm{p}>0,05$ )

\section{Discusión}

Rev. cienc. cuidad. 2020;17(3):33-45 
Con relación a la situación laboral se encontró que la mayoría de los egresados del programa de enfermería permanecen en Colombia y en la región Caribe; casi un $70 \%$ de graduados está trabajando, de los cuales, la mayoría tiene vinculación laboral en modalidad de término fijo o por orden de prestación de servicios y principalmente en el sector privado. La Universidad, como medio para conseguir trabajo, fue reconocida en un porcentaje bajo. La mayoría de quienes se encontraban trabajando al momento de la encuesta, reconocieron que su actividad laboral se relaciona directamente con el programa de formación. En ese sentido, la importancia de que los profesionales formados permanezcan en los territorios es relevante para reducir la escasez crítica de profesionales en enfermería, contribuyendo a mejorar la condición de la salud de las poblaciones (11).

El tema del empleo, de la posibilidad de encontrar trabajo al terminar la formación, se encuentra a lo largo del pregrado como un tema que genera ansiedad. Según un estudio realizado en Chile, algunos estudiantes han referido que la mayor preocupación es la de enfrentar dificultades para encontrar trabajo, por diferentes situaciones personales y por las experiencias de los egresados que dicen demorarse mucho tiempo en encontrar empleo (12). Esta situación es real, como se identificó en este estudio, donde algunos (12\%) se han demorado más de un año para conseguir trabajo. La situación del empleo enfermero es compleja en varias partes del mundo. Un estudio realizado en España, expuso la preocupación por llegar a tener tasas de desempleo superiores al $15 \%$; el análisis por años, reporto que los egresados del periodo de 2011 a 2014 aportaron enormemente al desempleo, lo cual descendió ligeramente en 2015, aumentando de nuevo en 2016 y 2017 (13).

La crisis laboral de la enfermería y de otras profesiones de salud, supera la respuesta de los programas académicos Según análisis realizado por la Asociación Nacional de Enfermeras de Colombia (ANEC), aspectos como el conflicto bélico, el cambio epidemiológico, el aumento de la pobreza, las crisis financieras y otros aspectos estructurales, han impactado el papel de la profesión de enfermería, afectando su empleabilidad (14).

Según los datos del Observatorio Laboral para la Educación (15), en el departamento del Magdalena, durante el periodo de 2007 a 2017, el segundo programa de salud con más graduados fue enfermería. Lo cual se contrasta con lo reportado por el Observatorio Laboral y Ocupacional Colombiano (16), en el cual dentro de las primeras 20 ocupaciones con mayor demanda laboral en el departamento no encuentra la enfermería.

A pesar de lo anterior, es evidente el déficit de profesionales de enfermería en Colombia. Según la Organización para la Cooperación y el Desarrollo Económico (OCDE), para el 2015, el índice de 1,1 profesionales de enfermería por cada 1.000 habitantes en Colombia, ubicó al país en el último lugar en razón a que el promedio mundial, para este grupo de países, fue de 9,9 por 1000 habitantes; el primer lugar lo tuvo Suiza con 18 por 1000 habitantes (17). Para 2018 se encontró que esa relación aumentó mínimamente, ubicando a Colombia en el rango entre 10-19 enfermeros por 1000 (18). Este dato evidencia la necesidad de sensibilizar e informar más sobre la importancia de la enfermería, para que la sociedad comprenda la relevancia de contar con suficientes profesionales de enfermería en diferentes áreas, más allá de lo clínico hospitalario, como en promoción de la salud, prevención de la enfermedad, salud comunitaria y familiar, entre otros.

Un estudio realizado en Canadá exploró los factores que afectan la satisfacción de los estudiantes de educación superior, las percepciones sobre su éxito académico y las expectativas futuras de empleo; encontrando que quienes sintieron mayor satisfacción con su desempeño académico y personal se sentían más preparados para enfrentar el mudo laboral (19). Aspecto fundamental a tener en cuenta por los programas de pregrado, sobre todo por la necesidad de mejorar la formación integral, incluyendo aspectos que permitan a los estudiantes sentirse seguros y confiados durante su formación profesional y prepararlos para la salida al mundo del trabajo de forma contextualizada. En Australia existe una estrategia que ha mejorado la preparación para el nuevo empleo en los estudiantes de pregrado de enfermería, que consiste en comprender el concepto de empleabilidad, incorporando las habilidades y competencias clínicas separadas del ámbito académico. A la fecha ha logrado reducir la presión y el estrés al terminar la formación familiarizándolos con el mundo del trabajo (20).

Continuando con la situación laboral, el tema del emprendimiento cobra importancia. Al respecto, se encontró que solamente algunos (12\%) egresados han constituido o tienen pro- 
yectos de emprendimiento. Con relación a este aspecto, una revisión sistemática encontró que las actitudes empresariales se encuentran principalmente en los enfermeros jóvenes y que a medida que aumenta la edad y el tiempo de ejercicio profesional se reduce el interés por el emprendimiento (21), lo cual indica que es fundamental continuar estimulando la generación de emprendimientos, desde el ejercicio liberal de la enfermería, orientados a la solución de problemas locales desde la formación de pregrado.

Con relación al impacto social, los enfermeros y enfermeras participantes, reconocieron que su formación ha sido de un buen nivel y se sienten, en su mayoría, satisfechos por los estudios realizados, por lo cual recomiendan el programa. Sin embargo, pocos se encuentran agremiados y/o vinculados con organizaciones académicas y científicas. Hasta la fecha de corte un $34 \%$ ha adelantado formación pos-gradual, un porcentaje bajo maneja otros idiomas y un $13 \%$ ha recibido reconocimientos. Al respecto, se hace necesario analizar la incorporación de competencias que promuevan la habilidad para ampliar y perfeccionar el desempeño profesional al hacer estudios postgraduales y de autoformación. Como lo propone la Escuela de Enfermería y Ciencias de la Salud de Tottori en Japón, considerándola fundamental para mejorar la calidad del cuidado y garantizar el desarrollo profesional (22). El estímulo de capacidades organizativas entre estudiantes de pregrado puede generar una conexión que favorezca pasar de la educación superior al mundo laboral (23), ya que al tener la perspectiva de cuerpo organizado, agremiado o sindicalizado, los egresados cuentan con una red de soporte y apoyo que favorece su cambio del rol estudiante al rol de trabajador.

La satisfacción es un aspecto que requiere indagaciones más profundas ya que se relaciona con múltiples aspectos de la vida. Un estudio realizado en Nepal reportó un $38,6 \%$ de satisfacción laboral entre los enfermeros (24); otro realizado en Estados Unidos reporto niveles de satisfacción académica que oscilaron entre el 56 y el 63\% (25) y uno en México encontró un $47 \%$ de satisfacción con la formación recibida (26), proporciones inferiores a la encontrada en el presente estudio.

Las posibilidades de tener un trabajo con apoyo de los compañeros, buenas relaciones de mando-subordinación, reconocimiento social y salarios justos son algunos de los aspectos que de- finen la satisfacción laboral $(27,28)$. Desde esa perspectiva se hace necesario impactar desde la Universidad, como lo han propuesto algunos autores, para sensibilizar a las organizaciones de salud y facilitar la inclusión de los nuevos profesionales, la mayoría millenials y centenials, incorporando procesos de ingreso graduales y amigables, que garanticen la acogida de estas nuevas generaciones de enfermeros y por ende que favorezcan la satisfacción de los mismos (29). Pero este proceso debe ir acompañado del impacto gremial o asociativo para lograr el pago justo al trabajo enfermero, aspecto fundamental para lograr la satisfacción de los profesionales más jóvenes (30).

Finalmente, en un contexto como Santa Marta o el Caribe en general, donde el turismo es una de las principales actividades económicas, el inglés se constituye en un aspecto que puede favorecer mayores opciones de vinculación laboral (31), así como una mejor atención a los pacientes de otros países. Al respecto, para el 2014 se estableció la meta de que un $20 \%$ de graduandos de los programas de enfermería a nivel nacional debía demostrar un nivel intermedio o superior de inglés, solo el 2,4 \% alcanzó el nivel B+ ese año (32). Igual a los resultados obtenidos en este estudio donde se encontró una proporción baja de egresados bilingües. Pero además, el inglés es un idioma requerido para la investigación, sobre todo para las búsquedas efectivas de artículos científicos (33), por lo tanto, se considera un aspecto que debe ser fomentado y fortalecido significativamente en la formación de pregrado.

\section{Conclusiones}

- La mayoría de los graduados han permanecido en el país y se encuentran vinculados laboralmente, sin embargo hay un porcentaje importante que refirió no estar trabajando, lo le genera un reto al programa en términos de gestionar opciones y políticas que estimulen la contratación de profesionales de enfermería.

- A pesar de las formas de vinculación laboral que no garantizan el pleno ejercicio de la profesión, un alto porcentaje se siente satisfecho o muy satisfecho con su formación recibida. Los egresados reconocen que el programa de enfermería de la Universidad del Magdalena tiene un currículo interesante, llamativo, y adecuado que, se ha adaptado a las demandas de la sociedad. Sin embargo, algunos aspectos del contexto se transforman a velocidades superiores a las posibilidades 
del programa y esto demanda nuevos retos.

- Los aspectos considerados relevantes para adaptar el programa son: estimular el emprendimiento para el ejercicio liberal de la profesión; diversificar con mayor ahínco los énfasis de la formación más allá de lo clínico hospitalario que le otorguen al graduado un perfil más diverso y con mayores posibilidades de vinculación en diferentes sectores de la sociedad; generar conexiones o puentes con empresas para favorecer el tránsito del graduado al mundo laboral, más allá de la práctica clínica; y estimular el el aprendizaje temprano de idiomas y el fortalecimiento de competencias de investigación. caron las propias de los estudios transversales con participación voluntaria que no garantiza el poder del dato para hacer inferencias a todos los graduados. Si bien los datos no pueden ser extrapolables a todos los egresados, si garantizan la calidad y veracidad de la información a partir de preguntas que permitieron el doble chequeo de los datos, para la información sociodemográfica, laboral y la calidad de la formación recibida, específicamente.

\section{Conflicto de Intereses}

Los autores declaran no tener ningún conflicto de intereses.

Como limitantes del este estudio se identifi-

\section{Referencias Bibliográficas}

1. González G, Díez-Echavarría L, Zapa E, Eusse D. Evaluación de impacto académico y social en egresados universitarios. Revista Complutense de Educación. 2019; 30 (3): 695-712.

2. Ramos-Duharte D, Robinson-Jay F, de-la-Caridad-Gómez Lloga T, Legrá-Terrero M, Mustelier-Vera K. El enfoque ciencia-tecnología-sociedad y la innovación social en los profesionales de la salud. Rev Inf Cient. 2017; 96(4):626-635.

3. De Arco-Canoles O del C, Suarez-Calle ZK. Rol de los profesionales de enfermería en el sistema de salud colombiano. Univ. Salud. 2018; 20(2):171-182. http://dx.doi.org/10.22267/ rus. 182002.121

4. Parra Castrillón JE, Arias-Giraldo SM. Metodología para caracterización y análisis de impacto en el medio de egresados de Instituciones de Educación Superior. Cooperativismo \& Desarrollo. 2017; 25(111):0-0. https://doi.org/10.16925/co.v25i111.1875

5. Herrera-Velázquez MR, Calderón-Macías ML, Zambrano-Santos RO, Placencia-López BM. Acreditación y evaluación de los procesos en la carrera de Enfermería en la Universidad Estatal del Sur de Manabí. Educ Med Super [Internet]. 2018 Dic [citado 2020 Ene 15]; 32(4):108120. Disponible en: http://scielo.sld.cu/scielo.php?script=sci arttext\&pid=S086421412018000400011\&lng=es

6. Salas F. Los estudios de seguimiento de la población graduada como herramienta para el cambio y la innovación en el currículo universitario: la experiencia de la Escuela de Administración Educativa de la Universidad de Costa Rica. Rev Educ. 2006; 30(2):63-81.

7. Colombia. Lineamientos para la acreditación de programas. CNA. Bogotá, Colombia, diciembre de 2013. https://www.mineducacion.gov.co/1759/articles-341938 archivo_pdf.pdf.

8. Republica de Colombia. Consejo Nacional de Educación Superior. Acuerdo 01 de 2018, Por el cual se actualizan lineamientos para acreditación de alta calidad institucional y de programas de pregrado [Internet] Bogotá DC 2018. [citado 2020 Ene 15] Disponible en: https:// www.mineducacion.gov.co/1759/articles-373938_recurso_1.pdf.

9. Valencia A, Lopera M, Arango CM, Segura AM. Impacto laboral, económico y social de los graduados de la Universidad CES; 2016. Rev CES Salud Pública. 2017; 8(2): 13-24. 
10. Álvarez-Hernández G, Delgado-DelaMora J. Diseño de Estudios Epidemiológicos. El Estudio Transversal: Tomando una Fotografía de la Salud y la Enfermedad. Bol Clin Hosp Infant Edo Son [Internet]. 2015 [citado 2020 Mayo 7]; 32(1):26-34. Disponible en: https://www. medigraphic.com/pdfs/bolclinhosinfson/bis-2015/bis151f.pdf

11. Anderson M, McKee C, Talbot T. Investing UK Aid in a Global Skills Partnership: Better Health at Home and Abroad. CGD Policy Paper [Internet]. Washington, DC: Center for Global Development. 2017. Disponible en: https:/www.cgdev.org/publication/investing-uk-aid-global-skills-partnershipbetter-health-home-and-abroad.

12. Vergara M, Gallardo G. ¿Cómo encontraré trabajo? Proyecciones imaginadas de transición al mundo del trabajo de estudiantes de pregrado. Psicoperspectivas. 2019; 18(3):1-12. http:// dx.doi.org/10.5027/psicoperspectivas-vol18-issue3-fulltext-1676

13. Sindicato de Enfermería. Análisis del empleo en enfermería Sindicato de Enfermería. [Documento en Internet]. Disponible en: http:/www.actasanitaria.com/wp-content/ uploads/2016/03/analisis-del-empleo-en-enfermeria.pdf

14. Carvallo-Suárez B, Criado-Morales ML. La Asociación Nacional de Enfermeras de Colombia en Yopal Casanare. universalud.rev.ciencias salud Unisangil 2015; 5(1):58-62. http://publicaciones.unisangil.edu.co/index.php/revista-universalud/article/view/311/278

15. Ministerio de Educación Nacional. Bases de datos. Observatorio Laboral para la Educación. Disponible en: http://bi.mineducacion.gov.co:8380/eportal/web/men-observatorio-laboral/ perfil-nacional

16. Aplicativo WEB de la Agencia Pública de Empleo. Cálculo Observatorio Laboral y Ocupacional -OLO. Cifras regionales del mercado laboral. Tendencias ocupacionales. Información actualizada de la tendencia de las ocupaciones que corresponde al año 2018. Disponible en: https://observatorio.sena.edu.co/Mercado/TendenciaOcupaciones.

17. OECD. "Nurses", in Health at a Glance 2017: OECD Indicators, OECD Publishing, Paris. 2017. Disponible en: https://doi.org/10.1787/health_glance-2017-56-en.

18. Organización Mundial de la Salud. Situación de la Enfermería en el mundo 2020. Resumen de Orientación [Internet]. OMS; 2020. Disponible en: https://apps.who.int/iris/bitstream/ handle/10665/331675/9789240003392-spa.pdf

19. James M, Yun D. Exploring student satisfaction and future employment intentions: A case study examination: is there a link between satisfaction and getting a job? Higher Education, Skills and Work-Based Learning. 2018; 8(2):117-133. https://doi.org/10.1108/HESWBL-03-2017-0019

20. Meller N. The Job Readiness initiative: a supportive approach to enhance nursing students' new graduate employability through a self-directed technology rich learning environment. Abstracts Teaching And Learning Conference Anz 2019, 20-22 August 2019. Sydney. 7-7. Retrieved from https://bbtlcanz.bbworld.com/wp-content/uploads/sites/6/2019/08/TLCANZ19_Program_V19.pdf

21. Da-Silva-Copelli FH, Lorenzini-Erdmann A, Guedes-dos-Santos JL. Entrepreneurship in Nursing: an integrative literature review. Rev. Bras. Enferm. 2019 Feb; 72(Suppl 1):289298. https://doi.org/10.1590/0034-7167-2017-0523

22. Fukada M. Nursing Competency: Definition, Structure and Development. Yonago Acta Medica 2018; 61:001-007. https://doi.org/10.33160/yam.2018.03.001

23. Elken M, Tellmann SM. Linking higher education and the world of work: learning outcomes and intermediary organisations. Journal of Education and Work. 
2019;32(8):678-692. https://doi.org/10.1080/13639080.2019.1696951

24. Sapkota A, Poudel UK, Pokharel J, Ghimire P, Sedhain A, Bhattarai G, et al. Factors associated with job satisfaction among graduate nursing faculties in Nepal. BMC Nurs. 2019; 18(58): https://doi.org/10.1186/s12912-019-0379-2

25. Hopkins J, Bromley G. Preparing New Graduates for Interprofessional Teamwork: Effectiveness of a Nurse Residency Program. J Contin Educ Nurs. 2016;47(3):140-148. https://doi. org/10.3928/00220124-20160218-10

26. Pecina-Leyva R. Satisfacción académica del estudiante de enfermería en una universidad pública en México. Revista Electrónica sobre Tecnología, Educación y Sociedad. 2017; 4(7):114. http://www.ctes.org.mx/index.php/ctes/article/view/639/697

27. Orgambídez-Ramos A, de-Almeida H. Work engagement, social support, and job satisfaction in Portuguese nursing staff: A winning combination. Appl Nurs Res. 2017 Aug 1;36:37-41. https://doi.org/10.1016/j.apnr.2017.05.012

28. Silva-Ribeiro da V, Velasque-de-Souza L, Tonini T. Job satisfaction in an oncology nursing team. Rev. Bras. Enferm. 2017 Oct; 70(5): 988-995. https://doi.org/10.1590/0034-71672016-0422

29. Hofler L. Transition of New Graduate Nurses to the Workforce. Challenges and Solutions in the Changing Health Care Environment. North Carolina Medical Journal. March-April 2016; 77(2): 133-136. http://doi:10.18043/ncm.77.2.133

30. Yew SY, Yong CC, Tey NP, Cheong KC, Ng ST. Work satisfaction among nurses in a private hospital. Int J Healthc Manag. 2018 Jun 21;1-8. https://doi.org/10.1080/20 $\underline{479700.2018 .1489459}$

31. San-Lucas-Marcillo M, Azua-Menéndez M, Tigua-Anzules J. El Idioma Inglés como segunda lengua en los profesionales de la carrera de enfermería. Revista Científica Sinapsis. 2017: 1(6):1-11. https://doi.org/10.37117/s.v1i6.60

32. Alonso-Cifuentes JC, Estrada-Nates D, Mueces-Bedón BV. Evaluación del nivel de inglés en los programas de enfermería en Colombia: 2011-2016. Revista Colombiana De Enfermería. 2019; 18(2);e009. https://doi.org/10.18270/rce.v18i2.2669

33. Meza-Fiallos CE, Robalino-Araujo MC. Aplicación del idioma ingles en la resolución de casos clínicos desde la perspectiva de la medicina basada en evidencia. Rev Cuba Reumatol [Internet]. 2019 Ago [citado 2020 Abr 07]; 21(2): e81. http://scielo.sld.cu/scielo.php?scrip$\mathrm{t}=$ sci_arttext\&pid=S1817-59962019000200004 